\title{
Trait-based approach to bacterial growth efficiency
}

$4 \quad{ }^{2}$ Current Address: Département des Sciences Biologiques, Université du Quebéc à Montréal, Montréal, Quebec, Canada

6

$8 \quad *$ Corresponding author, Tel 812-856-0962, Fax 812-855-6082, Email lennonj@indiana.edu

10 Running Title: Growth Efficiency Traits

12 Key Words: Bacterial Production, Bacterial Respiration, Physiological Trade-offs 


\section{Abstract}

Bacterial growth efficiency (BGE) is the proportion of assimilated carbon that is converted

18 into biomass and reflects the balance between growth and energetic demands. Often measured as an aggregate property of the community, BGE is highly variable within and across ecosystems.

20 To understand this variation, we focused on how species and resource identity affect BGE using a trait-based approach with 20 bacterial isolates enriched from north temperate lakes. We used

22 phenotypic and genomic approaches to characterize the metabolism of each isolate and test for predicted trade-offs between growth rate and efficiency. Regardless of resource identity, $20 \%$ of

24 the variation in BGE could be attributed to the taxonomic class of the bacterial species, and $58 \%$ of the variation could be explained by species identity. Meanwhile, resource identity explained a

26 relatively small amount of variation (7\%) in BGE across species but accounted for $>60 \%$ of the variation within a species. Metabolic trade-offs and genomic features suggest that BGE is a

28 predictable trait that is independent of resource type. As such, genomic and phylogenetic information from microbiomes may help predict aggregate community functions like BGE and

30 the fate carbon in ecosystems. 


\section{INTRODUCTION}

In most ecosystems, heterotrophic bacteria play a pivotal role in determining whether

36 organic carbon is respired as $\mathrm{CO}_{2}$ or converted into biomass and retained in food webs (Pomeroy et al. 1998; Bardgett et al. 2008; Ducklow 2008). Many factors control how bacteria process

38 carbon, but perhaps the most important is reflected by bacterial growth efficiency (BGE). BGE is the proportion of assimilated organic carbon that is converted into bacterial biomass (del Giorgio

40 and Cole 1998). When BGE is high, more carbon is turned into bacterial biomass where it can be retained for longer periods of time while also serving as a resource for other members of the food

42 web. In contrast, when BGE is low, microbially assimilated carbon has a shorter residence time and is released into the environment as $\mathrm{CO}_{2}$. When measured at the community scale, $\mathrm{BGE}$ is

44 notoriously variable among habitats and has proven difficult to predict (del Giorgio and Cole 1998).

While BGE can be influenced by a range of chemical and physical properties (Apple and del Giorgio 2007; Hall and Cotner 2007; del Giorgio and Newell 2012; Sinsabaugh et al. 2013;

48 Geyer et al. 2016), it may also reflect taxon-specific differences in microbial metabolism. For example, BGE results from the physiological balance between cellular growth and energetic

50 demands. As such, bacterial growth strategy is predicted to constrain BGE via physiological trade-offs (Litchman et al. 2015), and it has been hypothesized that oligotrophs have higher

52 maximum growth efficiency than copiotrophs (Roller and Schmidt 2015). In addition, species that specialize on only a few resources are predicted to be more efficient at using those resources

54 than generalist species (Dykhuizen and Davies 1980; Glasser 1984). As such, traits such as maximum growth rate and the number of resources used (i.e., niche breadth) could underlie 56 species-specific differences in BGE. 
Variation in BGE may also be influenced by the properties of resources that are used to

58 meet energetic and growth demands. Different resources can affect cellular ATP yield depending on the metabolic pathways used (Fuhrer et al. 2005; Flamholz et al. 2013). For example, glucose

60 is metabolized via glycolysis, but growth on aromatic compounds, such as protocatechuate, requires the $\beta$-ketoadipate pathway which yields less ATP (Gottschalk 1986). Furthermore,

62 energy-producing catabolic processes and biomass-producing anabolic processes are not independent (Russell and Cook 1995; Kempes et al. 2012). For example, cells have the potential

64 to produce > 30 ATP from a single glucose molecule if it is completely oxidized, but there would be no remaining carbon to yield new biomass. Instead, cells must use the intermediate products

66 of glycolysis to form proteins and other cellular material, which diminishes the maximum ATP yield (Gottschalk 1986; Flamholz et al. 2013). In addition, biomass production requires materials

68 and energy. For example, the synthesis of proteins, which constitute $\sim 70 \%$ of cellular dry mass, requires amino acid building blocks and 4 ATP per peptide bond (Tempest and Neijssel 1984;

70 Gottschalk 1986; Lynch and Marinov 2015). Therefore, because resources differ in their potential energy yield and bacteria differ in their ability to extract energy and form biomass from

72 a given resource, BGE should vary based on the resources available to bacteria.

In this study we measured BGE in a set of bacterial isolates growing on three different

74 carbon resources that varied in chemical structure and metabolic pathway (Fig. 1). In addition to partitioning variation in BGE based on species and resource identity, we tested for hypothesized

76 trade-offs with growth rate and niche breadth. Furthermore, using the genomes of each isolate, we evaluated whether metabolic pathways could explain differences in BGE among diverse

78 representatives of aquatic bacteria from north temperate lakes. Last, to test if resources have differential effects on the metabolic traits that underlie BGE (i.e., production and respiration), we 
80 tested for resource-specific relationships between respiration and production rates for each resource. Together, our trait-based approach provides a framework for understanding linkages

82 between community structure and function due to the physiological constraints on BGE and suggest that large changes in community composition or available resources may alter BGE and

84 therefore carbon cycling in predictive ways.

\section{RESULTS}

88 Bacterial Growth Efficiency - Using measures of bacterial productivity (BP) and respiration (BR), we calculated bacterial growth efficiency (BGE) for 20 isolates growing on three different

90 resources: glucose, succinate, and protocatechuate (Fig. 1). All isolates belonged to the Proteobacteria phylum with representatives from the Alpha-, Beta-, and Gammaproteobacteria

92 subphyla (Fig. 2, Fig. S1). Across isolates, we found that BGE ranged from <0.01 to 0.32 (Fig. 2). Based on linear mixed-effects models we found that isolate and resource identity explained

94 substantial amount of variation in BGE. Across resources, isolate identity explained $58 \%$ of the variation in BGE, and $67 \%$ of the variation within resource, and the taxonomic order of each

96 isolate explained $20 \%$ of the variation in BGE across resources, and $28 \%$ of the variation within resource. Across isolates, resource identity only explained $7 \%$ of the variation in BGE across all 98 isolates, but $63 \%$ of variation within isolate.

Next, we determined if there was any evidence of phylogenetic signal for BGE among

100 our isolates. Based on Blomberg's K (a measure of phylogenetic signal strength), there was weak phylogenetic signal for BGE. We did not detect phylogenetic signal for BGE when isolates used 102 succinate $(\mathrm{K}=0.002, p=0.24)$ or protocatechuate $(\mathrm{K}=0.001, p=0.146)$, but there was a 
significant phylogenetic signal when isolates used glucose $(\mathrm{K}=0.002, p=0.04)$. However, the

104 low K value suggests that BGE is over-dispersed (i.e., less phylogenetic signal than expected under Brownian motion). Furthermore, when we use Pagel's $\lambda$ (a measure of phylogenetic signal compared to Brownian motion), we found no evidence of a signal (Glucose: $\lambda=0.10, p=0.76$; Succinate: $\lambda=0.13, p=0.66$; Protocatechuate: $\lambda=<0.01, p=0.99)$. unimodal. Based on Hartigan's dip test, we found that there was a bimodal distribution of BGE 110 among our isolates $(\mathrm{D}=0.07, p=0.58$, Fig. S2). Using this distribution, we split isolates into two groups, which we define as the "high BGE", and "low BGE" groups.

Phenotypic Comparisons - Using linear models, we found phenotypic differences between

114 isolates that were related to BGE (Fig. 3). While there was no relationship between BGE and maximum growth rate $\left(\mu_{\max } ; \mathrm{F}_{1,7}=0.035, \mathrm{r}^{2}<0.01, p=0.86\right)$ in the low-BGE group of bacteria, 116 we identified a significant negative relationship between BGE and $\mu_{\max }$ for the high-BGE group $\left(\mathrm{F}_{1,7}=7.79, \mathrm{r}^{2}=0.53, p=0.027\right)$. Based on this model we would predict a $2.6 \%$ decrease in

118 BGE for each per minute increase in $\mu_{\max }$ in the high-BGE group. In contrast to our predictions, there was no relationship between niche breadth (Levin's Index) and BGE for the low-BGE 120 group $\left(\mathrm{F}_{1,7}=1.42, \mathrm{r}^{2}=0.17, p=0.27\right)$ or the high-BGE group $\left(\mathrm{F}_{1,7}=0.92, \mathrm{r}^{2}=0.11, p=0.37\right)$.

122 Genomic Comparisons - We found genomic differences between isolates that were related to BGE. First, isolates in the high-BGE group had $26 \%$ more annotated metabolic pathways (based 124 on an $80 \%$ module completion ratio cutoff) than isolates in the low BGE-group $\left(\mathrm{m}_{\text {high }}=66\right.$, $\mathrm{m}_{\text {low }}=652$, $\mathrm{t}$-test: $\left.\mathrm{t}_{18}=-2.64, p=0.02\right)$. Second, we found that the number of metabolic 
126 pathways was related to the observed BGE when grown on glucose, but the relationship depended on the group. For the high-BGE group there was a negative relationship between BGE

128 and the number of pathways $(\beta=-0.006, \mathrm{r} 2=0.48, p=0.04)$, but for the low-BGE group there was a positive relationship $(\beta=0.0003, \mathrm{r} 2=0.37, p=0.05)$. Next, we found that differences in

130 the metabolic pathway composition of a genome could help explain which group an isolate belongs, and pathway composition was related to the observed value of BGE. Specifically, we

132 found three pathways that were indicators of an isolate being in the high-BGE group (Table 1). Likewise, for the high-BGE group we found that we could explain $24 \%$ of the variation in BGE

134 based on the composition of metabolic pathways (dbRDA: $\left.\mathrm{F}_{1,7}=2.17, \mathrm{R}^{2}=0.24, p=0.05\right)$. We found eight pathways with significant positive or negative correlations $(|\rho|>0.7)$ with BGE

136 (Table 2). However, we not find a relationship between pathway composition and BGE for the low-BGE group $(p=0.45)$.

Resource Effects - Indicator variable linear regression revealed a positive relationship between

140 respiration and production rates $\left(\mathrm{Fig} .4, \mathrm{~F}_{9,42}=8.07, \mathrm{R}^{2}=0.63, p<0.001\right)$ with there being a higher $y$-intercept for the high-BGE group of isolates $\left(\beta_{\text {Group }}=2.7, p<0.001\right)$. Resource identity,

142 however, had no effect on the BR-BP relationship or the effect of group (all $p>0.25$ ). Last, we did not find evidence that the slope of the BR-BP relationship was different from one $\left(t_{42}=0.76\right.$,

$144 p=0.45)$; therefore, the two measures of bacterial metabolism appear to scale proportionately with one another.

\section{DISCUSSION}


148 We measured bacterial growth efficiency (BGE) in 20 environmental bacterial isolates grown on three resources that varied in their bioavailability, structure, and pathways required for metabolism (Fig. 1). While BGE varied across isolates, there was no evidence for a strong phylogenetic signal in efficiency. However, a substantial amount $(20 \%)$ of the variation in BGE

152 could be explained by an isolate's taxonomic order while a much smaller amount of the variation (7\%) could be attributed to resource identity (Fig. 3). We found evidence for the predicted trade-

154 off between maximum growth rate and efficiency, but only on the most labile resource (glucose) (Fig. 3). Even though we found that resource identity explained $63 \%$ of the variation in BGE

156 within an isolate, resource identity did not alter the relationship between respiration and production rate observed across isolates (Fig. 4) suggesting that resource identity has a stronger

158 effect on BGE within a species than across species. Together, we propose that growth efficiency is a physiological trait independent of resource identity, but resource characteristics may modify

160 species-specific physiological performances. We propose that taxonomic groups of bacteria may have fundamentally different growth efficiencies such that changes in community composition

162 may alter the fate of carbon resources (i.e., biomass versus $\mathrm{CO}_{2}$ ) within the ecosystem.

\section{Bacterial Growth Efficiency as a Trait}

Our results indicate that there are species-specific properties regulating BGE, which may be conserved at higher taxonomic levels. This conclusion is consistent with the view that BGE represents a complex bacterial trait (i.e., aggregate property of numerous cellular functions) with 168 ecological significance, and that different groups of bacteria have fundamentally different strategies for carbon allocation. Our phylogenetic analyses suggest that BGE may be an over170 dispersed trait (at least with glucose) such that the efficiency of closely related bacteria may be 
less similar than expected. One potential explanation for this pattern of over-dispersion is that

172 our culture collection lacked phylogenetic resolution within some of our taxonomic groups (e.g., Betaproteobacteria) or that the variation in BGE within a taxonomic group (e.g., order) may not

174 be the same across taxonomic groups. Alternatively, BGE may not be a phylogenetically conserved trait. Though some traits such as phosphorus acquisition, photosynthesis, and

176 methanogenesis are phylogenetically conserved deep in the microbial tree of life (Martiny et al. 2006, 2013), others such as complex carbon metabolism are not (Zimmerman et al. 2013).

178 Therefore, it is possible that BGE may be similar to traits such as complex carbon metabolism that are not deeply conserved, which appears to be common among complex traits (Martiny et al. 2015). Regardless, our data reveal that BGE is a complex bacterial trait that is influenced by taxonomic affiliation. As such, it may be possible to make predictions about BGE and other

182 ecosystem functions given information about composition of resident microbiomes (Goberna and Verdú 2016).

\section{Bacterial Growth Efficiency on Different Resources}

186 Differences in resource complexity and the metabolic pathways required for degradation may explain species-specific differences in BGE due to resource identity. Within an isolate, resource

188 identity accounted for $63 \%$ of the variation in BGE. Given that different resources are processed via different metabolic pathways, resource-based variation in BGE within a species is expected.

190 For example, BGE was higher when isolates were supplied with glucose compared to when they were supplied with protocatechuate. Glucose is a simple sugar that is able to be metabolized by

192 numerous pathways and converted to acetyl-CoA (Neidhardt 2007). Protocatechuate, on the other hand, is a complex aromatic compound that requires a specific metabolic pathway to be 
194 converted to acetyl-CoA. Furthermore, because protocatechuate is chemically more complex, it requires more energy (i.e., ATP) to be degraded than more labile resources such as glucose

196 (Harwood and Parales 1996). Therefore, resource complexity and the metabolic pathways required may explain the within-isolate variation in BGE. However, across isolates, we did not

198 find resource-specific differences in the relationship between respiration and production rate. Such findings suggest that energetic demands required to use different resources may also be

200 species-specific trait. That is, the energetic demands for individual species may be highly constrained and therefore not change much when growing on different resources. Together, these

202 findings suggest that the effect of resources on the efficiency of entire microbiomes may depend on the composition of bacteria consuming those resources.

\section{Bacterial Growth Efficiency Groups}

Across all isolates, we found a bimodal distribution of BGE suggesting that there were two distinct groups with contrasting efficiencies. One group had low BGE $(<5 \%)$ across all

208 treatments, and the other group ranged in BGE from 7-30 \% (Fig. $2 \&$ 3). Although, the range of BGE measured across isolates is similar to the range observed in many ecosystems (del Giorgio

210 and Cole 1998), our results suggest that some species of bacteria grow relatively inefficiently, irrespective of resource quality. One explanation is that the minimum cellular energetic demand

212 (i.e., cellular maintenance costs) is higher in some bacteria than others (Russell and Cook 1995). Furthermore, energetic demand may be higher when bacteria are grown in minimal media where

214 they must produce all cellular components from a single carbon resource (Tao et al. 1999). Alternatively, nutrient concentrations (e.g., phosphorus) and other physical properties (e.g., 216 temperature) may regulate efficiency (Smith and Prairie 2004; Frey et al. 2013) and the effects of 
these properties may be species-specific. As such, it is possible that maintenance costs, resource

218 imbalances, and the physical growth conditions affected BGE of our isolates. Differences in lowBGE and high-BGE isolates was also reflected in genomic content, including the number and

220 presence-absence of metabolic pathways. Together, these findings suggest that there are fundamental differences between bacterial species that determine BGE. However, these

222 genomic features seem to best explain large-scale rather than fine-scale differences in BGE.

\section{Physiological Trade-Offs}

We found evidence to support a trade-off between maximum growth rate and BGE (Fig. 4),

226 which is predicted in microbial and non-microbial systems (Glasser 1984; Roller and Schmidt 2015). For example, theoretical models of microbial communities predict a rate-efficiency trade-

228 off (Allison 2014), and this trade-off has been observed across microbial taxa (Lipson 2015).

Physiologically, the trade-off is based on allocation constraints imposed by the balance between

230 energy requirements and biomass yield: organisms with higher maximum growth rates may have more energetic requirements and thus lower BGE (Russell and Baldwin 1979; Russell and Cook

232 1995). Furthermore, processes that limit respiration, such as oxygen availability, have been shown to suppress bacterial growth rate (Meyenburg and Andersen 1980). Therefore, respiration

234 rate is likely a major control on biomass production and BGE. Consistent with this, we observed a scaling relationship between respiration and production rates (Fig. 4). Furthermore, this

236 relationship between respiration and production and the non-zero intercept suggest that there is a minimum respiration rate required before any biomass can be produced, which is commonly

238 interpreted as the cellular maintenance requirement. Therefore, it is possible that the maintenance 
energy demand of bacterial species explains the physiological trade-off between maximum

240 growth rate and growth efficiency.

Theory also predicts a trade-off between resource niche-breadth and growth efficiency

242 (Glasser 1984). This trade-off is based on the assumption that there is an energetic cost to maintaining numerous metabolic pathways (Johnson et al. 2012). As such, species with more

244 metabolic pathways should have more energetic requirements and thus lower BGE; although, the effects of genome reduction has been debated (Giovannoni et al. 2005; Livermore et al. 2014). In

246 this study, we did not find evidence of a trade-off between resource niche breadth and BGE (Fig. 4). One possible explanation is that the resources used in our phenotypic assay (i.e. Ecolog

248 plates) did not reflect the full metabolic potential of our isolates. Alternatively, there may not be a strong trade-off between niche breadth and efficiency, but further experiments with additional

250 isolates and resources would be required to test this prediction more rigorously.

\section{Genomic Signatures}

In addition to the physiological differences documented among our isolates, we found genomic

254 evidence of metabolic pathways that are associated with BGE. Specifically, we found genomic differences between isolates that belong to low-BGE and high-BGE groups. We discovered that

256 isolates in the high-BGE group had $26 \%$ more metabolic pathways than the low-BGE group.

Furthermore, we identified three pathways that were unique to the high-BGE group (Table 1)

258 and a number of pathways that were correlated with the observed BGE (Table 2). Together, our findings suggest that there are genomic features that may contribute to or regulate BGE.

260 In general, the genomic composition of BGE groups appear to reflect differences in cellular biosynthesis. It is possible that species with particular biosynthesis pathways may generate 
262 essential cellular components with less energetic demand. For example, the low-BGE isolates lacked some metabolic pathways, including pyridoxal biosynthesis and histidine degradation,

264 which were present in the high-BGE group. The pyridoxal biosynthesis pathway produces vitamin $B_{6}$ from erythrose-4-phosphate (Mukherjee et al. 2011). Because vitamin $B_{6}$ is essential

266 for growth, the isolates lacking the pyridoxal pathway must use alternatives such as uptake from the environment if they are auxotrophic (i.e., unable to synthesize) or other synthesis pathways

268 such as the Deoxyxylulose-5-phosphate synthase (DXS) pathway (Mukherjee et al. 2011). However, the DXS pathway requires pyruvate (a precursor for Krebs cycle) and thus may limit

270 central metabolism and possibly lead to lower BGE. Likewise, the histidine degradation pathway is used to breakdown histidine into ammonium and glutamate (Bender 2012). Alternatively,

272 glutamate can by synthesized from $\alpha$-ketoglutarate; however, because $\alpha$-ketoglutarate is an intermediate component of Krebs cycle this may limit central metabolism and possibly lead to

274 reduced BGE.

\section{Conclusion}

At the cellular level, BGE reflects the balancing energetic and cellular growth demands. We find evidence of this based on physiological trade-offs (i.e., maximum growth rate) as well

278 as metabolic pathways. As such, changes in community composition and resource availability have to potential to alter food web and ecosystem function due to changes in BGE. For example,

280 communities dominated by species with low BGE should yield a net release of $\mathrm{CO}_{2}$ from the ecosystem. Alternatively, communities comprised of individuals with high BGE should yield a

282 net increase in ecosystem productivity. However, variation in BGE can arise within a species due to the ways in which it processes different resources. Therefore, changes in the resource supply 284 will alter the performance of individual taxa, but we predict that these changes will not be as 

286 based approach can be used to provide a mechanistic link between the structure and function of bacterial communities.

strong as changes in BGE that arise owing to differences in community composition. A trait-

\section{METHODS}

290 Bacterial Isolates - Using a novel cultivation approach, we isolated 20 bacterial strains from lakes in the Huron Mountain Research Preserve (Powell, MI, USA) by incubating inert carbon

292 beads (Bio-Sep Beads) in the water column for one week. Prior to the incubations, the beads were saturated with a sterile complex carbon substrate, i.e., Super Hume (CropMaster, United

294 Agricultural Services of America, Lake Panasoffkee, Florida, USA). Super Hume is a lignin-rich resource comprising $17 \%$ humic and $13 \%$ fulvic acids, and has been shown to be an analog of

296 terrestrial DOC in aquatic ecosystems that can be used by diverse bacteria (Lennon et al. 2013).

We used this enrichment technique to select for bacteria with a range of metabolic potentials

298 (Ghosh et al. 2009). After the incubation, beads were rolled on R2 agar plates (BD Difco, Sparks Maryland, USA) and incubated at $25^{\circ} \mathrm{C}$. We picked random colonies from plates and serially

300 transferred until axenic. All isolates were preserved in $25 \%$ glycerol at $-80{ }^{\circ} \mathrm{C}$.

We identified each bacterial strain by direct sequencing the 16S rRNA gene. We obtained

302 genomic DNA from log phase cultures using the FastPrep DNA extraction kit according to the manufacturer's specifications (MP Biomedical). We used $10 \mathrm{ng}$ of genomic DNA to amplify the

304 16S rRNA gene using the 27F and 1492R bacterial primers (See Supplemental for primer sequences and PCR conditions). We sequenced the PCR products at the Indiana Molecular

306 Biology Institute (IMBI) at Indiana University (Bloomington, Indiana, USA). Raw sequence reads were quality-trimmed based on a Phred quality score of 25. Forward and reverse reads 
were manually merged after aligning sequences to the Silva 16S SSU rRNA reference alignment (release 132) using SINA v. 1.2.11 and the Bacteria variability profile (Pruesse 2011). After

310 merging into full length 16S rRNA sequences, we used mothur (Schloss et al. 2009) to check the quality of sequences and alignments were checked using the ARB software package (Ludwig et

312 al. 2004). Finally, sequences were compared to the Silva All-Species Living Tree Project database (Yilmaz et al. 2014) for taxonomic identification (Fig. S1).

Bacterial Growth Efficiency — To test for differences in bacterial growth efficiency (BGE), we

316 measured BGE for each isolate when grown on each of three different carbon substrates: glucose, succinate, or protocatechuate (Fig. 2). These carbon sources (i.e., resources) were

318 chosen based on differences in their bioavailability and structure but also the required pathways for metabolism (see Fig. 2).

To determine BGE we measured bacterial respiration and production rates and then calculated $\mathrm{BGE}$ as $\mathrm{BP} /(\mathrm{BP}+\mathrm{BR})$, where $\mathrm{BP}$ is bacterial productivity and $\mathrm{BR}$ is bacterial

322 respiration (del Giorgio and Cole 1998). BP and BR were measured using triplicate cultures of each isolate. Cultures of each isolate were grown in R2 broth (BD Difco, Sparks Maryland,

324 USA) until mid-log phase. We then transferred $100 \mu \mathrm{L}$ of culture into $10 \mathrm{~mL}$ of M9 broth (Green and Sambrook 2012) with the appropriate carbon source (25 mM C) and allowed $24 \mathrm{~h}$ for the

326 cultures to acclimate. We then transferred $100 \mu \mathrm{L}$ of culture into $10 \mathrm{~mL}$ of fresh carbon-amended M9 broth and incubated 1-3 h to replenish nutrients. Using these transfers, we were able to 328 establish populations of each isolate at target cell densities between $10^{4}$ and $10^{5}$ cells $\mathrm{mL}^{-1}$. We used the populations to measure BP and BR, which were normalized to cell density using plate 330 counts of colony forming units. We measured BP using the ${ }^{3} \mathrm{H}$-Leucine assay (Smith and Azam 
1992) with $1.5 \mathrm{~mL}$ of culture. We added ${ }^{3} \mathrm{H}$-Leucine to a final concentration of $50 \mathrm{mM}$ and

332 incubated for $1 \mathrm{~h}$. Following incubation, we terminated production with trichloroacetic acid (final concentration $3 \mathrm{mM}$ ) and measured leucine incorporation using a liquid scintillation

334 counter. We measured BR using an automated $\mathrm{O}_{2}$ measurement system (PreSens Sensor Dish System, PreSens, Regensburg, Germany) on $5 \mathrm{~mL}$ of culture based on the rate $\mathrm{O}_{2}$ consumption

336 during three-hour incubations. We estimated BR as the slope of $\mathrm{O}_{2}$ concentration during the incubation using linear regression. We used theoretical respiratory quotients for each resource to 338 convert $\mathrm{O}_{2}$ depletion into $\mathrm{C}$ respiration assuming aerobic growth.

340 Taxonomic and Phylogenetic Relationships - We compared differences in BGE across isolates and resources using linear models. First, we used a taxonomic framework to compare

342 BGE between isolates (Lennon et al. 2012). Isolates were classified into taxonomic groups based on the species tree constructed in ARB. We then used mixed linear models to compare BGE

344 across taxonomic groups and resources. To test the hypothesis that taxonomy (i.e., at the class level) affects BGE, we nested resource identity within isolate in the linear model. To test the

346 hypothesis that resource identity affects BGE, we nested isolate identity within resource. We identified the best statistical models based on the variation explained $\left(\mathrm{R}^{2}\right)$ and AIC values.

348 Second, we tested if phylogenetic relationships between isolates explained differences in BGE across isolates. We created a phylogenetic tree based on the full-length 16S rRNA gene

350 sequences. We aligned sequences using the SINA aligner (Pruesse et al. 2012) and checked alignments using ARB. We generated a phylogenetic tree using the CIPRES science gateway

352 (Miller et al. 2010). The phylogenetic tree was created using RAxML v.8.2.12 (Stamatakis 2006). We used the GTRGAMMA DNA substitution model and the rapid hill-climbing 
354 algorithm to build our maximum likelihood trees, and we used the extended majority rule to find the consensus tree. We used Blomberg's K and Pagel's Lambda to compare trait variation across

356 the tree and test if phylogenetic relationships between isolates could explain differences in traits (Pagel 1999; Blomberg et al. 2003). Blomberg's K is a test for phylogenetic signal that

358 determines if trait variation is better explained by phylogenetic relationships or Brownian motion. Pagel's Lambda is a test of phylogenetic signal that determines if trait variation differs

360 from Brownian motion. Last, to determine if the distribution of BGE across isolates was unimodal, we used Hartigan's dip test for unimodality (Hartigan and Hartigan 1985). Hartigan's

362 dip test is used to determine if a distribution is unimodal by testing the null hypothesis that there is a dip in the distribution. A significant Hartigan's dip test would suggest that the distribution is

364 unimodal. Alternatively, the distribution has an internal "dip" (reported as D). All statistical tests were conducted in the R statistical environment (R Core Development Team 2013). We used the 366 nlme package (Pinheiro and Bates 2011) for the mixed linear models, the picante package (Kembel et al. 2015) for the phylogenetic methods, and the diptest package (Maechler 2015) for 368 Hartigan's dip test.

370 Phenotypic Comparisons and Trade-offs - To test the hypothesis that phenotypic differences and physiological trade-offs underlie BGE variation, we compared the maximum growth rate

372 ( $\mu$ max) and niche breadth of each isolate. First, to test whether BGE was affected by growth strategy (i.e., copiotrophs vs. oligotrophs), we measured the maximum growth rate of each

374 isolate. Bacterial growth rates were measured based on changes in optical density during 18-h incubations. Bacterial isolates were grown in R2 broth in 48-well plates. We incubated plates with continuous shaking and measured optical density every 15 min using a plate reader (BioTek 
MX). Growth curves were analyzed by fitting a modified Gompertz growth model (Zwietering et

378 al. 1990; Lennon 2007) to the observed growth curves using maximum likelihood fitting. We used the model fit as our estimate of $\mu$ max.

Second, to test whether BGE was affected by niche breadth, we generated carbon usage profiles using BioLog EcoPlates ${ }^{\mathrm{TM}}$ (Garland and Mills 1991). The EcoPlate is a phenotypic

382 profiling tool consisting of 31 unique carbon sources. In addition to the carbon source, each well contains a tetrazolium dye, which in the presence of NADH will be reduced and change color.

384 We used this colorimetric assay to generate carbon usage profiles for each strain. We standardized profiles for each strain by subtracting water blanks (average water blank +1 SD), and relativizing across substrates. Using these data, we calculated resource niche breadth using Levin's Index (Colwell and Futuyma 1971).

Genomic Comparisons - To test the hypothesis that variation in metabolic pathways could

390 explain differences in BGE, we compared the genomes of each isolate. First, we determined the metabolic pathways found in the genome of each isolate. We characterized each isolate using

392 whole genome sequencing. Genomic DNA libraries for each isolate were prepared using the Illumina TruSeq DNA sample prep kit using an insert size of 250 base pairs (bp). Libraries were 394 sequenced on an Illimina HiSeq 2500 (Illumina, San Diego, GA) using 100-bp paired-end reads at the Michigan State University Research Technology Support Facility. We processed raw 396 sequence reads (FASTQ) by removing the Illumina TruSeq adaptors using Cutadapt (Martin 2011), interleaving reads using Khmer (McDonald and Brown 2013), and quality-filtering based 398 on an average Phred score of 30 using the FASTX-toolkit (Hannon Lab 2010). Finally, we normalized coverage to 25 based on a k-mer size of 25 using Khmer. We assembled the genomes 
400 using Velvet (Zerbino and Birney 2008) after optimizing assembly parameters for each isolate with Velvet Optimizer (Gladman and Seemann 2012). We annotated contigs larger than $200 \mathrm{bp}$

402 using Prokka (Seemann 2014), and predicted metabolic and physiological functions using MAPLE with bidirectional best-hit matches (Takami et al. 2012). We identified functional 404 pathway based on the presence of intermediate genes within a pathway. We scored pathways as functional if more than $80 \%$ of the intermediate genes were recovered in the genomes based on 406 module completion ratios.

To test the hypothesis that metabolic pathways affect BGE, we used multivariate methods

408 to compare the pathways of each isolate. First, we used PERMANOVA to determine if there were differences in pathways associated with different levels of BGE. When significant

410 differences were found, we used indicator species analysis (Dufrene and Legendre 1997) to determine which metabolic pathways contributed to group differences in BGE. Next, to

412 determine if metabolic pathways could explain differences in BGE within a group, we used distance-based redundancy analysis (dbRDA) which is a multivariate technique that tests if a

414 quantitative predictor can explain differences in multivariate datasets (Legendre and Legendre 2012). Because we scored pathways as present or absent, metabolic distances between isolates 416 were calculated using the Jaccard Index. We tested for significance using a permutation test. If the dbRDA model was significant, we used Spearman's rank-order correlation to test for 418 correlations between BGE and individual metabolic pathways. We used the vegan R package (Oksanen et al. 2012) for multivariate analyses.

Resource Effects - To test the hypothesis that resources have different effects on components 422 of metabolism that affect BGE, we used a linear model to test for a relationship between BR and 
BP. Because BP required energy through respiration, we used production rate as the dependent

424 variable and respiration rate as the independent variable. We used an indicator variable linear regression to test for changes in BP rate due to BR. We included resource identity and group

426 (high- versus low-BGE) as the categorical predictors and BR as the continuous predictor (Lennon and Pfaff 2005). In addition, we included all interactions terms. Respiration and

428 production rates were $\log _{10}$-transformed to meet model assumptions. Last, to determine if the relationship between BR and BP rates was isometric (proportional scaling, slope $=$ one) or

430 allometric (disproportional scaling, slope $\neq$ one), we used a one-sample t-test to determine if the slope was different from one. All statistical tests were conducted in the R statistical environment.

\section{ACKNOWLEDGEMENTS}

434 We thank BK Lehmkuhl and MA Carrison for technical assistance and JB McKinlay and members of the Lennon Lab for critical feedback on an earlier version of this manuscript. This

436 work was supported by the Huron Mountain Wildlife Foundation (MEM \& JTL), the National Science Foundation (DEB-0842441, DEB-1442246, and DEB-1501164), and US Army Research

438 Office (W911NF-14-1-0411). All code and data used in this study can be found in a public GitHub repository (https://www.github.com/LennonLab/MicrobialCarbonTraits). Isolate 440 genomes are available on NCBI (BioProject PRJNA420393). 


\section{REFERENCES}

444 Allison, S. D. 2014. Modeling adaptation of carbon use efficiency in microbial communities. Frontiers in Microbiology 5:1-9.

446 Apple, J. K., and P. A. del Giorgio. 2007. Organic substrate quality as the link between bacterioplankton carbon demand and growth efficiency in a temperate salt-marsh estuary. The 448 ISME Journal 1:729-742.

Bardgett, R. D., C. Freeman, and N. J. Ostle. 2008. Microbial contributions to climate change

450 through carbon cycle feedbacks. The ISME Journal 2:805-14.

Bender, R. A. 2012. Regulation of the histidine utilization (Hut) system in bacteria.

452 Microbiology and Molecular Biology Reviews 76:565-584.

Blomberg, S. P., T. Garland, and A. R. Ives. 2003. Testing for phylogenetic signal in

454 comparative data: behavioral traits are more labile. Evolution 57:717-745.

Buchan, A., L. S. Collier, and E. L. Neidle. 2000. Key aromatic-ring-cleaving enzyme,

456 protocatechuate 3,4-dioxygenase, in the ecologically important marine Roseobacter lineage. Applied and Environmental Microbiology 66:4662-4672.

458 Colwell, R. K., and D. J. Futuyma. 1971. On the Measurement of Niche Breadth and Overlap. Ecology 52:567-576.

460 del Giorgio, P. A., and J. J. Cole. 1998. Bacterial growth efficiency in natural aquatic systems. Annual Review of Ecology and Systematics 29:503-541.

462 del Giorgio, P. A., and R. E. I. Newell. 2012. Phosphorus and DOC availability influence the partitioning between bacterioplankton production and respiration in tidal marsh ecosystems.

464 Environmental Microbiology 14:1296-307.

Ducklow, H. W. 2008. Bacterial Production and Biomass in the Oceans. Pages 1-47 inMicrobial 
Ecology of the Oceans.

Dufrene, M., and P. Legendre. 1997. Species assemblages and indicator species: the need for a

468 flexible asymmetrical approach. Ecological Monographs 67:345-366.

Dykhuizen, D., and M. Davies. 1980. An Experimental Model: Bacterial Specialists and

470 Generalists Competing in Chemostats. Ecology 61:1213-1227.

Flamholz, A., E. Noor, A. Bar-Even, W. Liebermeister, and R. Milo. 2013. Glycolytic strategy as

472 a tradeoff between energy yield and protein cost. Proceedings of the National Academy of

Sciences 110:10039-44.

474 Frey, S. D., J. Lee, J. M. Melillo, and J. Six. 2013. The temperature response of soil microbial efficiency and its feedback to climate. Nature Climate Change 3:395-398.

476 Fuhrer, T., E. Fischer, and U. Sauer. 2005. Experimental identification and quantification of glucose metabolism in seven bacterial species. Journal of Bacteriology 187:1581-1590.

478 Garland, J. L., and A. L. Mills. 1991. Classification and characterization of heterotrophic microbial communities on the basis of patterns of community-level sole-carbon-source

480 utilization. Applied and Environmental Microbiology 57:2351-9.

Geyer, K. M., E. Kyker-Snowman, A. S. Grandy, and S. D. Frey. 2016. Microbial carbon use

482 efficiency: accounting for population, community, and ecosystem-scale controls over the fate of metabolized organic matter. Biogeochemistry 127:173-188.

484 Ghosh, D., K. Roy, V. Srinivasan, T. Mueller, O. H. Tuovinen, K. Sublette, A. Peacock, et al. 2009. In-situ enrichment and analysis of atrazine-degrading microbial communities using

486 atrazine-containing porous beads. Soil Biology and Biochemistry 41:1331-1334.

Giovannoni, S. J., H. J. Tripp, S. Givan, M. Podar, K. L. Vergin, D. Baptista, L. Bibbs, et al. 488 2005. Genome streamlining in a cosmopolitan oceanic bacterium. Science 309:1242-5. 
Gladman, S., and T. Seemann. 2012. Velvet Optimizer.

490 Glasser, J. W. J. 1984. Evolution of efficiencies and strategies of resource exploitation. Ecology 65:1570-1578.

492 Goberna, M., and M. Verdú. 2016. Predicting microbial traits with phylogenies. The ISME Journal 10:959-967.

494 Gottschalk, G. 1986. Bacterial Metabolism (2nd ed.). Springer-Verlag, New York, NY.

Green, M. R., and J. Sambrook. 2012. Molecular Cloning: A Laboratory Manual (4th ed.). Cold 496 Spring Harbor Laboratory Press, Cold Spring Harbor, New York.

Hall, E. K., and J. B. Cotner. 2007. Interactive effect of temperature and resources on carbon 498 cycling by freshwater bacterioplankton communities. Aquatic Microbial Ecology 49:35-45. Hannon Lab. 2010. FASTX Toolkit.

500 Hartigan, J. A., and P. M. Hartigan. 1985. The dip test of unimodality. The Annals of Statistics 13:70-84.

502 Harwood, C. S., and R. E. Parales. 1996. The beta-ketoadipate pathway and the biology of selfidentity. Annual Review of Microbiology 50:553-90.

504 Johnson, D. R., F. Goldschmidt, E. E. Lilja, and M. Ackermann. 2012. Metabolic specialization and the assembly of microbial communities. The ISME Journal 6:1985-1991.

506 Kembel, S. W., D. D. Ackerly, S. P. Blomberg, W. K. Cornwell, M. R. Helmus, M. Helene, and C. O. Webb. 2015. Picante: R tools for integrating phylogenies and ecology.

508 Kempes, C. P., S. Dutkiewicz, and M. J. Follows. 2012. Growth, metabolic partitioning, and the size of microorganisms. Proceedings of the National Academy of Sciences 109:495-500.

510 Legendre, P., and L. F. J. Legendre. 2012. Numerical Ecology (Vol. 24). Elsevier.

Lennon, J. T. 2007. Diversity and metabolism of marine bacteria cultivated on dissolved DNA. 
512 Applied and Environmental Microbiology 73:2799-2805.

Lennon, J. T., Z. T. Aanderud, B. K. Lehmkuhl, and D. R. J. Schoolmaster. 2012. Mapping the

514 niche space of soil microorganisms using taxonomy and traits. Ecology 93:1867-1879.

Lennon, J. T., S. K. Hamilton, M. E. Muscarella, A. S. Grandy, K. Wickings, and S. E. Jones.

516 2013. A source of terrestrial organic carbon to investigate the browning of aquatic ecosystems.

PloS One 8:e75771.

518 Lennon, J. T., and L. E. Pfaff. 2005. Source and supply of terrestrial organic matter affects aquatic microbial metabolism. Aquatic Microbial Ecology 39:107-119.

520 Lipson, D. A. 2015. The complex relationship between microbial growth rate and yield and its implications for ecosystem processes. Frontiers in Microbiology 6:1-5.

522 Litchman, E., K. F. Edwards, and C. A. Klausmeier. 2015. Microbial resource utilization traits and trade-offs: implications for community structure, functioning, and biogeochemical impacts at

524 present and in the future. Frontiers in Microbiology 06:1-10.

Livermore, J. A., S. J. Emrich, J. Tan, and S. E. Jones. 2014. Freshwater bacterial lifestyles

526 inferred from comparative genomics. Environmental Microbiology 16:746-758.

Ludwig, W., O. Strunk, R. Westram, L. Richter, H. Meier, Yadhukumar, A. Buchner, et al. 2004.

528 ARB: a software environment for sequence data. Nucleic Acids Research 32:1363-71.

Lynch, M., and G. K. Marinov. 2015. The bioenergetic costs of a gene. Proceedings of the

$530 \quad$ National Academy of Sciences 112:201514974.

Maechler, M. 2015. diptest: Hartigan's dip test statistic for unimodality.

532 Martin, M. 2011. Cutadapt removes adapter sequences from high-throughput sequencing reads.

EMBnet.journal 17:10.

534 Martiny, A. C., M. L. Coleman, and S. W. Chisholm. 2006. Phosphate acquisition genes in 
Prochlorococcus ecotypes: evidence for genome-wide adaptation. Proceedings of the National 536 Academy of Sciences 103:12552-7.

Martiny, A. C., K. Treseder, and G. Pusch. 2013. Phylogenetic conservatism of functional traits 538 in microorganisms. The ISME Journal 7:830-838.

Martiny, J. B. H., S. E. Jones, J. T. Lennon, and A. C. Martiny. 2015. Microbiomes in light of

540 traits: A phylogenetic perspective. Science 350:aac9323-aac9323.

McDonald, E., and C. T. Brown. 2013. Khmer: working with big data in bioinformatics. arXiv

$5421303.2223: 1-18$.

Meyenburg, K. V. O. N., and K. B. Andersen. 1980. Are growth rates of Escherichia coli in

544 batch cultures limited by respiration? Journal of Bacteriology 144:114-123.

Miller, M. A., W. Pfeiffer, and T. Schwartz. 2010. Creating the CIPRES Science Gateway for

546 inference of large phylogenetic trees. 2010 Gateway Computing Environments Workshop.

Mukherjee, T., J. Hanes, I. Tews, S. E. Ealick, and T. P. Begley. 2011. Pyridoxal phosphate:

548 biosynthesis and catabolism. Biochimica et Biophysica Acta - Proteins and Proteomics

1814:1585-1596.

550 Neidhardt, F. C. 2007. The Physiology and Biochemistry of Prokaryotes, 3rd ed. Microbe

Magazine (4th ed., Vol. 2). Oxford University Press, New York, NY.

552 Oksanen, A. J., F. G. Blanchet, R. Kindt, P. Legen-, P. R. Minchin, R. B. O. Hara, G. L.

Simpson, et al. 2012. Community Ecology Package. ... Ecology Package ....

554 Pagel, M. 1999. Inferring the historical patterns of biological evolution. Nature 401:877-884.

Pinheiro, J., and D. Bates. 2011. Linear and nonlinear mixed effects models (nlme). Package of

556 the R software for statistical computing on the CRAN repository.

Pomeroy, L. R., P. J. Williams, F. Azam, and J. E. Hobbie. 1998. The microbial loop. 
Oceanography 20:28-33.

Pruesse, E. 2011. SINA: high throughput multiple sequence alignment.

560 Pruesse, E., J. Peplies, and F. O. Glöckner. 2012. SINA: accurate high-throughput multiple sequence alignment of ribosomal RNA genes. Bioinformatics 28:1823-1829.

562 R Core Development Team. 2013. A language and environment for statistical computing.

Roller, B. R., and T. M. Schmidt. 2015. The physiology and ecological implications of efficient

564 growth. The ISME Journal 9:1481-1487.

Russell, J. B., and R. L. Baldwin. 1979. Comparison of maintenance energy expenditures and

566 growth yields among several rumen bacteria grown on continuous culture. Applied and Environmental Microbiology 37:537-543.

568 Russell, J. B., and G. M. Cook. 1995. Energetics of bacterial growth: balance of anabolic and catabolic reactions. Microbiological Reviews 59:48-62.

570 Schloss, P. D., S. L. Westcott, T. Ryabin, J. R. Hall, M. Hartmann, E. B. Hollister, R. A.

Lesniewski, et al. 2009. Introducing mothur: open-source, platform-independent, community-

572 supported software for describing and comparing microbial communities. Applied and Environmental Microbiology 75:7537-7541.

574 Seemann, T. 2014. Prokka: rapid prokaryotic genome annotation. Bioinformatics 30:2068-9. Sinsabaugh, R. L., S. Manzoni, D. L. Moorhead, and A. Richter. 2013. Carbon use efficiency of 576 microbial communities: stoichiometry, methodology and modelling. (J. Elser, ed.)Ecology Letters 16:930-939.

578 Smith, D. C., and F. Azam. 1992. A simple, economical method for measuring bacterial protein synthesis rates in seawater using 3H-leucine. Marine Microbial Food Webs 6:107-114.

580 Smith, E. M., and Y. T. Prairie. 2004. Bacterial metabolism and growth efficiency in lakes: the 
importance of phosphorus availability. Limnology and Oceanography 49:137-147.

582 Stamatakis, A. 2006. RAxML-VI-HPC: maximum likelihood-based phylogenetic analyses with thousands of taxa and mixed models. Bioinformatics 22:2688-2690.

584 Takami, H., T. Taniguchi, Y. Moriya, T. Kuwahara, M. Kanehisa, and S. Goto. 2012. Evaluation method for the potential functionome harbored in the genome and metagenome. BMC Genomics $586 \quad 13: 699$.

Tao, H., C. Bausch, C. Richmond, F. R. Blattner, and T. Conway. 1999. Functional genomics:

588 expression analysis of Escherichia coli growing on minimal and rich media. Journal of Bacteriology 181:6425-6440.

590 Tempest, D. W., and O. M. Neijssel. 1984. The status of YATP and maintenance energy as biologically interpretable phenomena. Annual Reviews in Microbiology 459-486.

592 Yilmaz, P., L. W. Parfrey, P. Yarza, J. Gerken, E. Pruesse, C. Quast, T. Schweer, et al. 2014. The SILVA and "All-species Living Tree Project (LTP)" taxonomic frameworks. Nucleic Acids 594 Research 42:643-648.

Zerbino, D. R., and E. Birney. 2008. Velvet: algorithms for de novo short read assembly using de 596 Bruijn graphs. Genome Research 18:821-9.

Zimmerman, A. E., A. C. Martiny, and S. D. Allison. 2013. Microdiversity of extracellular 598 enzyme genes among sequenced prokaryotic genomes. The ISME Journal 7:1187-1199.

Zwietering, M. H., I. Jongerburger, F. M. Rombouts, and K. van’t Riet. 1990. Modeling of the 600 bacterial growth curve. Applied and Environmental Microbiology 56:1875-1881. 


\section{TABLES}

604 Table 1: Genetic pathways unique to the high BGE isolates. Pathways are functional metabolic pathways identified from genome sequencing and predicted using Maple. Prob. = probability

606 statistic from indicator species analysis: the probability that the "species" (i.e., pathway) is not unique to the group.

\begin{tabular}{cccc}
\hline Group & Prob. & Pathway & Reference Function \\
\hline High BGE & 0.02 & M00045 & $\begin{array}{c}\text { Histidine degradation } \\
\text { (histidine } \rightarrow \text { N-formiminoglutamate } \rightarrow \text { glutamate) }\end{array}$ \\
& 0.03 & M00565 & $\begin{array}{c}\text { Lipopolysaccharide biosynthesis } \\
\text { (Kdo2-lipid A biosynthesis) }\end{array}$ \\
& & $\begin{array}{c}\text { Trehalose biosynthesis } \\
\text { (D-glucose-1P } \rightarrow \text { trehalose) }\end{array}$ \\
\hline
\end{tabular}

608 
612 Table 2: Genetic pathways correlated with BGE in the high-BGE group. Correlations are spearman's rank correlations between BGE and the pathway presence. Pathways with

614 correlations greater than 0.70 or less than -0.70 were considered significant.

\begin{tabular}{|c|c|c|c|}
\hline Relationship & $\rho$ & Pathway & Reference Function \\
\hline \multirow{2}{*}{ Positive } & 0.82 & M00025 & Tyrosine biosynthesis (chorismate $\rightarrow$ tyrosine) \\
\hline & 0.72 & M00034 & Methionine salvage pathway \\
\hline \multirow{6}{*}{ Negative } & -0.73 & M00117 & Ubiquinone biosynthesis (chorismate $\rightarrow$ ubiquinone) \\
\hline & -0.82 & M00044 & Tyrosine degradation (tyrosine $\rightarrow$ homogentisate) \\
\hline & -0.82 & M00053 & $\begin{array}{l}\text { Pyrimidine deoxyribonuleotide biosynthesis } \\
\text { (CDP/CTP } \rightarrow \text { dCDP/dCTP,dTDP/dTTP) }\end{array}$ \\
\hline & -0.82 & M00549 & Nucleotide sugar biosynthesis (glucose $\rightarrow$ UDP-glucose) \\
\hline & -0.82 & M00568 & Catechol ortho-cleavage (catechol $\rightarrow$ 3-oxoadipate) \\
\hline & -0.82 & M00637 & Anthranilate degradation (anthranilate $\rightarrow$ catechol) \\
\hline
\end{tabular}

616

618

620 
bioRxiv preprint doi: https://doi org/10.1101/427161 t this version posted January 20,2020 . The copyright holder for this preprint (which was not certified by peer review) is the author/funder, who has granted bioRxiv a license to display the preprint in perpetuity. It is made available under aCC-BY 4.0 International license. 


\section{FigURE LEGENDS}

626 Fig. 1: Carbon resources used to study BGE variation in environmental isolates. A: Glucose the baseline resource used to compare BGE across isolates. Glucose can be degraded by the

628 Embden-Meyerhof-Parnas, pentose phosphate, or Entner-Doudoroff pathway. Ultimately, these pathways produce pyruvate (and then acetyl-CoA), which enters Krebs cycle and is used to

630 produce energy and intermediate for biomass synthesis, when cells are grown aerobically.

Alternatively, glucose can be fermented into organic acids (e.g., lactate), but these reactions yield

632 less energy (Gottschalk 1986). B: Succinate - is a simple organic acid. Succinate is an

intermediate of Krebs cycle and thus it does not require previous degradation. Additionally,

634 succinate can be used directly to produce energy via succinate dehydrogenase (Neidhardt 2007).

C: Protocatecuate - is a complex resource with an aromatic core. Typically, it is degraded to

636 acetyl-CoA and Succinyl-CoA via the $\beta$-ketoadipate pathway (Harwood and Parales 1996).

Protocatechuate is commonly used to study aromatic resource degradation in ecosystems, and the

$638 \beta$-ketoadipate pathway is commonly found in bacteria across the phylum Proteobacteria (Buchan et al. 2000). 
642 Fig. 2: Bacterial growth efficiency (BGE) of each isolate for each resource. BGE was calculated based on measured production $(\mathrm{BP})$ and respiration $(\mathrm{BR})$ rates using the following equation:

$644 \mathrm{BGE}=\mathrm{BP} /(\mathrm{BP}+\mathrm{BR})$. Cladogram is based on the consensus phylogeny. Taxonomic class and order are included based on the ribosomal database taxonomy: $\alpha=$ Alphaproteobacteria. $\beta=$

646 Betaproteobacteria, $\gamma=$ Gammaproteobacteria, Xan. $=$ Xanthomonadales, Aero. $=$ Aeromondales, Pseu.$=$ Pseudomondales.

Fig. 3: Phenotypic traits associated with BGE. A: Maximum growth rate, a measure of growth 650 strategy, demonstrates a trade-off (negative relationship) with BGE in the high BGE group ( $\mathrm{F}_{1,7}$ $\left.=9.52, \mathrm{r}^{2}=0.54, p=0.015\right)$, but not the low BGE group $\left(\mathrm{F}_{1,7}=0.51, \mathrm{r}^{2}=0.06, p=0.50\right) . \mathrm{B}$ :

652 Levin's Index, a measure of niche breadth, does not demonstrate a trade-off with BGE in either the high or low BGE groups (high: $\mathrm{F}_{1,7}=0.92, \mathrm{r}^{2}=0.11, p=0.37$; low: $\mathrm{F}_{1,7}=1.47, \mathrm{r}^{2}=0.17, p=$

654 0.27). High and low BGE groups were determined based on bimodal distribution of BGE.

656 Fig. 4: Relationship between respiration and production rates. Respiration and production rates were compared using an indicator variable linear regression $\left(\mathrm{F}_{9,42}=4.92, \mathrm{R}^{2}=0.51, p<0.001\right)$.

658 According to the regression model, production rate increases with respiration rate proportionally (i.e., slope not significantly different from one, $t_{42}=-0.26, p=0.79$ ). In addition, group (high vs.

660 low BGE) was a significant factor and isolates in the high BGE group had a greater y-intercept $(p=0.02)$. Symbols indicates isolate group (high and low BGE), and color indicates the resource 662 being used. Symbol size is scaled by growth efficiency. 
bioRxiv preprint doi: https://doi.org/10.1101/427161; this version posted January 20, 2020. The copyright holder for this preprint (which was not certified by peer review) is the author/funder, who has granted bioRxiv a license to display the preprint in perpetuity. It is made available under aCC-BY 4.0 International license.

664

Figures

Fig. 1:

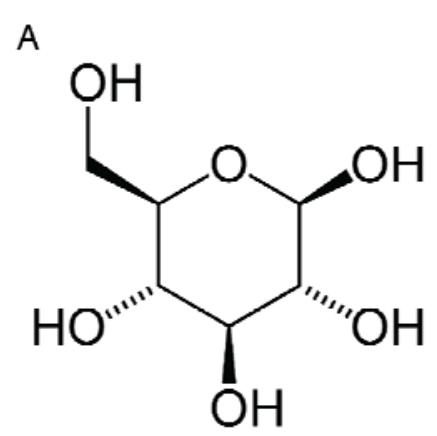

Glucose
B<smiles>O=C(O)CCC(=O)O</smiles>

Succinate<smiles>O=C(O)c1ccc(O)c(O)c1</smiles>

Protocatecuate 
bioRxiv preprint doi: https://doi.org/10.1101/427161· this version posted January 20,2020. The copyright holder for this preprint (which was not certified by peer review) is the author/funder, who has granted bioRxiv a license to display the preprint in perpetuity. It is made available under aCC-BY 4.0 International license.

668

Fig. 2:

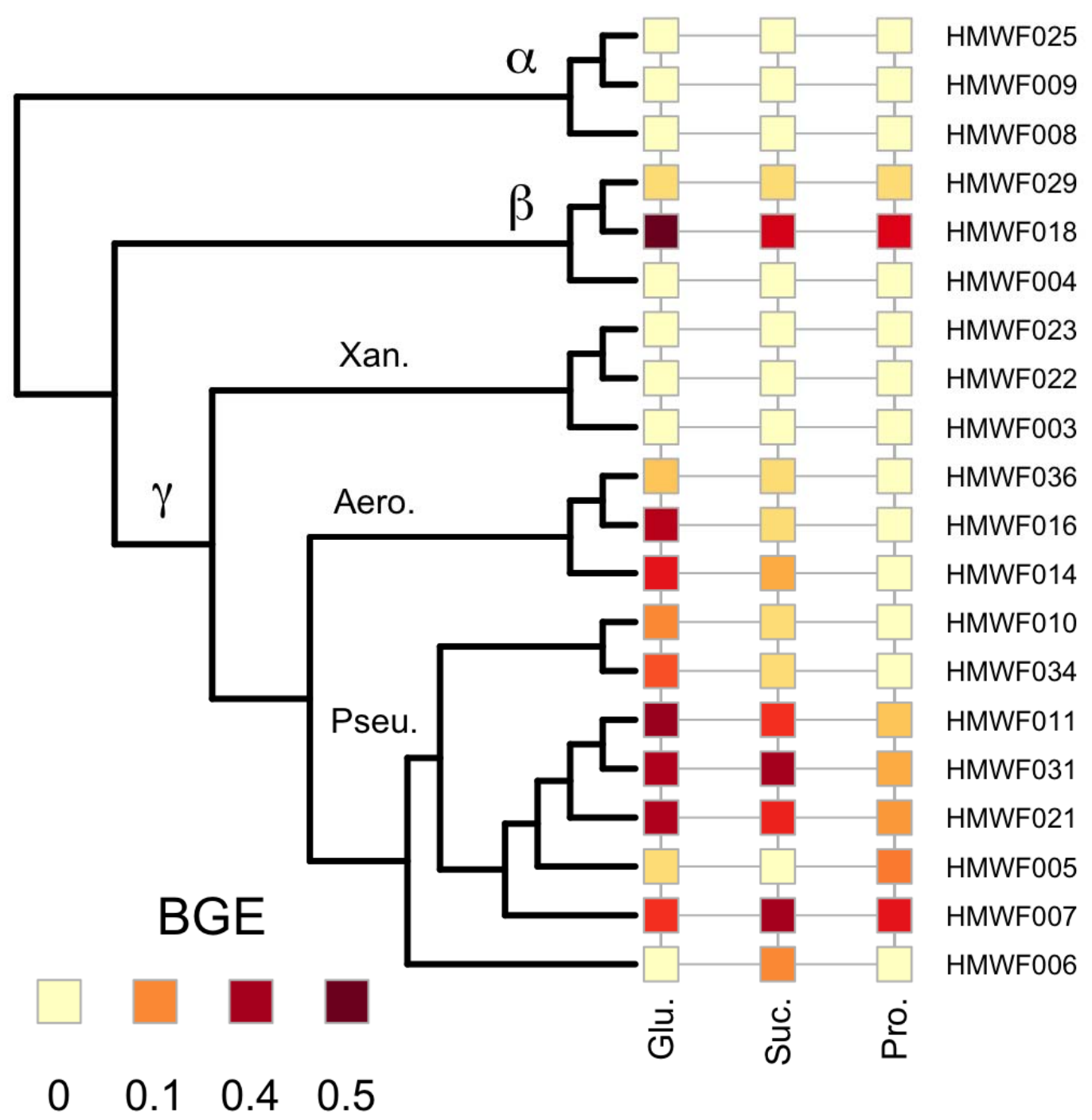


bioRxiv preprint doi: https://doi.org/10.1101/427161; this version posted January 20, 2020. The copyright holder for this preprint (which was not certified by peer review) is the author/funder, who has granted bioRxiv a license to display the preprint in perpetuity. It is made available under aCC-BY 4.0 International license.

672

Fig. 3:
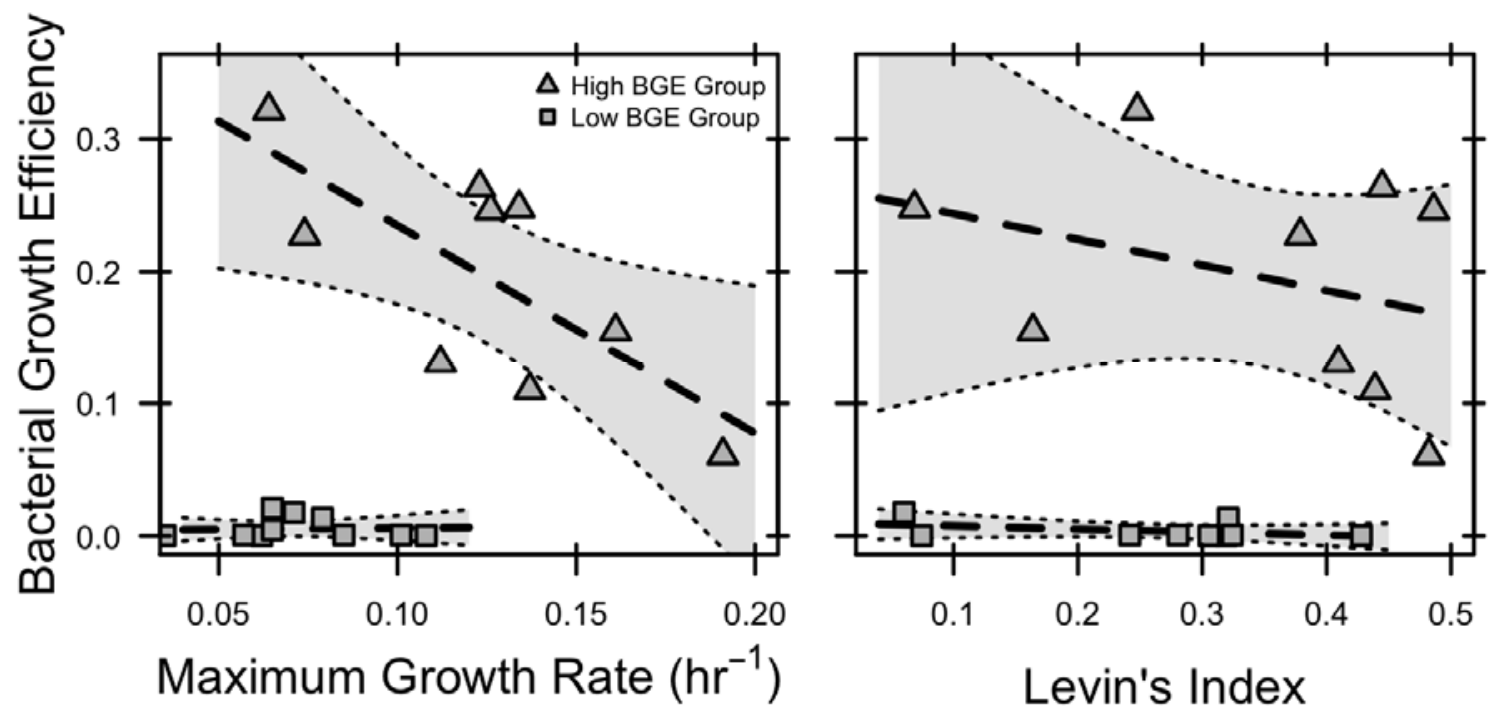

674 


\section{Fig. 4:}

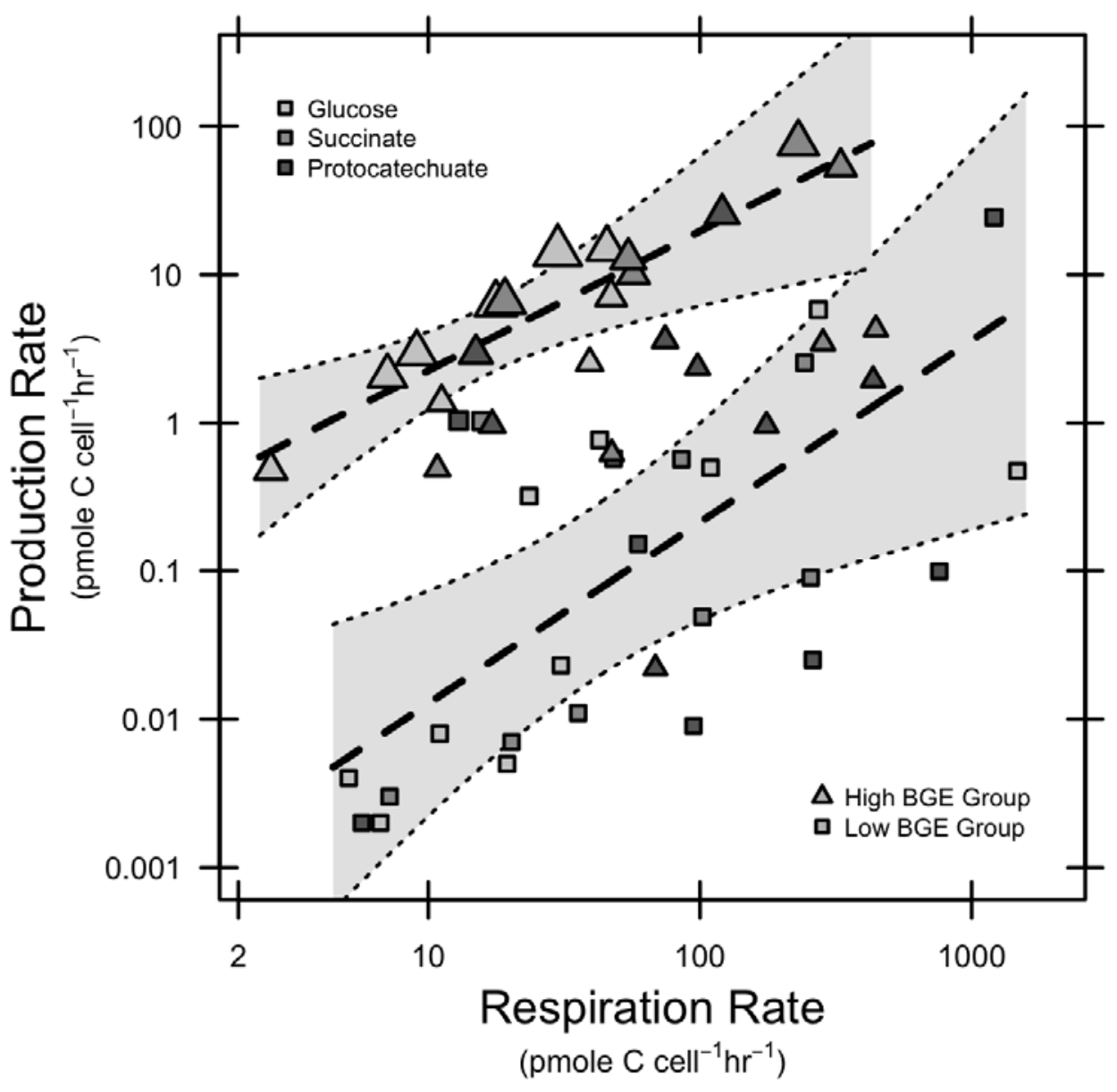

